\title{
Low revision rate and excellent subjective outcome of primary ACL repair with a minimum follow-up of 5
} years

\author{
Thomas $\mathrm{Nau}^{1,2,4}$ \\ Andreas Teuschl 2,3,4 \\ Anna Ebner ${ }^{1}$ \\ Ilse Jung ${ }^{2}$ \\ Christian Schenk ${ }^{1}$ \\ 1 Sanatorium Dr. Schenk GmbH, \\ Schruns, Austria \\ 2 Ludwig Boltzmann Institute of Experimental and \\ Clinical Traumatology, AUVA Research Center, \\ Vienna, Austria \\ 3 University of Applied Sciences, Technikum Wien, \\ Department of Chemical Engineering, \\ Vienna, Austria \\ 4 The Austrian Cluster for Tissue Regeneration, \\ Vienna, Austria
}

Corresponding author:

Thomas Nau

Ludwig Boltzmann Institute for Experimental

and Clinical Traumatology

Donaueschingenstrasse 13

1200 Vienna, Austria

Tel.: +436507273077

E-mail: thnau@hotmail.com

\section{Summary}

Introduction: Due to limitations of $\mathrm{ACL}$ reconstruction, primary $A C L$ repair has recently regained research interest. Although abandoned in the past, primary repair with conservation of the original ligament demonstrates considerable advantages compared to reconstruction.

We hypothesized that early repair, strictly limited to patients with a proximal $A C L$ rupture and excellent tissue quality of the remaining $A C L$ stump, would lead to equal revision rates and subjective outcomes as reported for ACL reconstruction after a minimum of 5 years.

Methods: In this questionnaire study, patients who had a primary ACL repair between 2002 and 2009 were invited to participate. Besides any potential revision surgery, the Tegner activity scale and the Knee Injury and Osteoarthritis Outcome Score were included in the evaluation.

Results: Out of 1912 patients who had ACL related surgery during the observation period, 221
(11.4\%) had a primary ACL repair. 60 patients $(61$ knees) were available for follow-up. In 2/61 (3.3\%) cases ACL revision surgery was performed and one patient had meniscus surgery of the affected side. The median Tegner activity scale was 6 (range, 3 to 10). The mean KOOS subscores were $\mathbf{8 8 . 8 \%}$ (Function/Sports), 86.6\% (Quality of life), 94.6 (Symptoms), 94.0 (Pain) and 97.0 (Activities of Daily Living).

Conclusion: Primary ACL repair, strictly limited to proximal ruptures with good tissue quality leads to revision rates and subjective outcome comparable to $A C L$ reconstruction.

Level of evidence: IV.

KEY WORDS: anterior cruciate ligament, outcome, sports injury.

\section{Introduction}

Due to discouraging results of clinical studies from the 1970 s and 1980 s, primary repair of the anterior cruciate ligament $(A C L)$ was more or less abandoned and replaced by surgical reconstruction with autograft or allograft material as the gold standard from the 1990s onwards ${ }^{1-5}$. The clinical results achieved by $A C L$ reconstruction are generally accepted. Nevertheless evident problems are well known, such as graft failure, donor site morbidity, loss of proprioception and the inexact anatomical form of reconstruction $^{6,7}$. In addition, despite subjective and objective joint stability following reconstruction, the risk for posttraumatic arthritis remains ${ }^{8-10}$.

Because of these ongoing problems, novel approaches to improve outcomes are developed. Only recently, primary $A C L$ repair has regained increasing research interest. A number of preclinical studies demonstrated the potential role of primary repair in combination with modern surgical techniques and enhancing bioactive materials ${ }^{7,11-13}$. Taylor et al. mentioned that despite the reported unacceptable high failure rates of previously published clinical studies, there constantly was a subset of patients that met the criteria for clinical success ${ }^{14}$. Proximal tears of the ACL, good tissue quality and early surgical repair were the main criteria in those patients that achieved satisfying results. In their thorough review, the Authors concluded that improved surgical techniques and appropriate patient selection may resurrect ACL primary repair as a realistic surgical option. 
In our institution, early surgical stabilization of $A C L$ tears, including primary repair, has a long tradition and is offered to the patients within 72 hours of injury. Based on the results of preoperative magnetic resonance imaging (MRI) and the findings during arthroscopy, a decision is made whether a primary repair or a surgical reconstruction of the ACL is performed. The option of primary repair is strictly limited to proximal tears and excellent tissue quality of the $\mathrm{ACL}$ stump.

It was the aim of the present study to show the revision rate and subjective outcome following primary ACL repair with a minimum follow-up of 5 years. We hypothesized that early repair, strictly limited to patients with a proximal $A C L$ rupture and excellent tissue quality of the remaining $A C L$ stump, would lead to equal revision rates and subjective outcomes as reported for ACL reconstruction. We also examined demographic factors, age and gender, regarding their influence on the different aspects of the reported outcome.

\section{Material and methods}

In this study which meets the ethical standard of this journals, using the institutional data base, all patients who received surgical treatment of an ACL injury between January 1, 2002, and December 31, 2009, were identified ${ }^{15}$. Only patients having a primary ACL repair as their initial surgical treatment within 72 hours after injury were considered. Patients with injuries to the posterior cruciate ligament, the lateral collateral ligament and/or the posterolateral corner were not included in this study. The decision to perform a primary repair in contrast to an ACL reconstruction was made by the surgeon based on the MRI appearance of the injured $A C L$ and on the findings during the arthroscopic examination (Fig. 1). The indication was strictly limited to proximal ruptures near the femoral insertion site with adequate integrity of the ligament stump.

\section{Surgical technique and postoperative protocol}

All surgeries were performed by the senior Author. Technically, the repair was performed arthroscopically. In general, two PDS suture loops were used to reattach the $A C L$ stump to its femoral insertion site. The sutures were stitched through the ACL stump in a $90^{\circ}$ angle to each other and then separately guided in an "over the top" direction towards the proximal lateral aspect of the knee joint. The knee was brought near extension, the sutures were tensioned and then tied over a bridge of the iliotibial band that was formed by two separate longitudinal incisions. The procedure was completed by microfracturing of the femoral insertion site of the ACL to stimulate healing response. The postoperative protocol allowed for immediate weight bearing and unrestricted range of motion as tolerated by the patient. In case of concomitant medial collateral ligament injury, brace immobilization for four weeks with free range of motion was

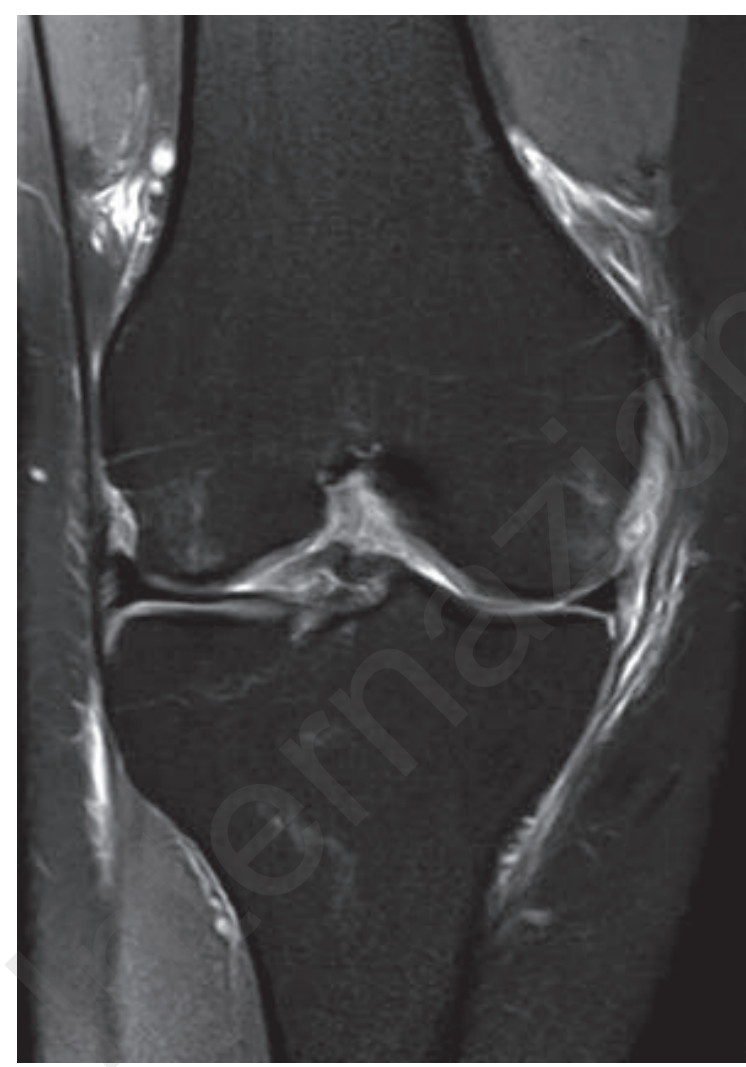

Figure 1. MRI demonstrates a proximal ACL tear. The ligament stump appears to be of adequate length and integrity. Additional arthroscopic examination of the remaining ligament will guide the decision making if a primary ACL repair is possible.

performed. Physiotherapy started immediately, cyclic activities were allowed after 6 weeks and high pivoting activities were prohibited for 4 months.

\section{Questionnaire}

The patients were contacted by mail between February 1 and April 30, 2015 and invited to participate in the study. After giving written consent, they were asked to complete a three part questionnaire:

Potential Revision Surgery: The answer was dichotomized (yes/no). Furthermore the time and the type of potential revision surgery was asked (ACL reconstruction, meniscus, other).

Tegner Activity Scale (TAS): The TAS is a standardized scale to grade work and sporting activities. The score varies from 0 to 10 . A score of 0 represents disability pension or sick leave because of knee problems, and a score of 10 correspondents to competing at national and international level ${ }^{16}$.

The Knee Injury and Osteoarthritis Outcome Score (KOOS): The KOOS evaluates knee-related function on five subscales: symptoms, pain, activities of daily living (ADL), function in sport and recreation (Function/Sports), and knee related quality of life (QoL). A subscore is calculated for each subscale, ranging from 0 (worst) to 100 (best) ${ }^{17}$. 


\section{Statistical analysis}

Mean and standard deviation as well as median were used for descriptive statistics. Data were tested for normal distribution. As all data were non-normal distributed, non parametric Mann Whitney U-test was used for gender comparison regarding TAS and KOOS subscores. A correlation analysis of TAS and KOOS subscores using Spearman's RHO test was performed to test for any correlation to age.

\section{Results}

A total of 1912 patients received surgical treatment of an ACL injury during the observation period. 1688 patients had an ACL reconstruction and were excluded from this analysis. 224 of these patients $(11.7 \%)$ had a primary ACL repair with the surgical technique as described above and met the inclusion criteria for this study. Out of this group, 60 patients (1 bilateral, 61 knees) were available for follow-up and completed the questionnaire, resulting in a response rate of $26.8 \%$. A detailed overview, including demographics and site of injury is presented in Table I.

$2 / 61(3.3 \%)$ cases had revision surgery of the primarily repaired $A C L$ and consequently received an $A C L$ reconstruction. In one case this was done 23 months after the initial ACL repair, in the other case, this was done after 35 months. One patient had additional meniscal surgery on the affected site, and another two patients answered "yes" because of unrelated surgery in other body regions during the follow-up period. The median TAS was 6, ranging from 3 to 10 . Figure 2 presents the results of the KOOS subscores. The mean subscores for "Function/Sports" and "Quality of Life" which are considered to be the most responsive after ACL surgery were $88.8 \%$, and $86.6 \%$ respectively. There was no significant gender related differences of TAS $(p=0.08)$ or of the KOOS $(p=0.82)$. Correlation analysis did not reveal any significant influence of age on the TAS ( $p=0.95)$ or on any of the KOOS subscores ( $p>0.05$ for all subscores).

\section{Discussion}

The results support the hypothesis that primary ACL repair, strictly limited to proximal ruptures and good tissue quality of the remaining ligament leads to revision rates and subjective outcome comparable to ACL reconstruction. Only 3.3\% (2/61) underwent revision surgery and consequent $A C L$ reconstruction during the observation period. Gifstad et al. reported on the revision rates of nearly 46.000 patients using the Scandinavian $A C L$ registries ${ }^{18}$. Five years after primary $A C L$ reconstruction, the revision rate was $2.8 \%$ for patellar tendon autografts and $4.2 \%$ for hamstring autografts. Although their registry based study primarily focused on the revision rates comparing patellar tendon and hamstring autografts, the large number of prospectively gathered patients definitely represent the results of the current gold standard in the treatment of $\mathrm{ACL}$ injuries. Other studies using $\mathrm{ACL}$ registries showed revision rates between 1.8 and $10.4 \%{ }^{19-22}$. In terms of subjective outcome, the KOOS subscores in the present series, all achieved comparable or higher scores to other ACL outcome studies with a similar observation period. Kvist et al. reported on the subjective results of the Swedish ACL registry using the $\mathrm{KOOS}^{23}$. Five years after primary $A C L$ reconstruction, the subscores ranged from 66 for "Quality of Life" to 69 for "Function/Sports". A further breakdown of their study regarding graft choice did not demonstrate any differences in the KOOS subscores. The registry based outcome studies, constantly revealed a higher revision rate of young com-

Table I. Demographic overview of patients available for 5-year minimum follow-up.

\begin{tabular}{|c|c|c|c|c|}
\hline & Male & in $\%$ & Female & in $\%$ \\
\hline 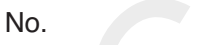 & 21 & 35 & 39 & 65 \\
\hline \multicolumn{5}{|l|}{ Site of surgery } \\
\hline left & 11 & 52 & 20 & 51 \\
\hline right & 9 & 43 & 19 & 49 \\
\hline bilateral & 1 & 5 & 0 & 0 \\
\hline \multicolumn{5}{|l|}{ Age at surgery } \\
\hline $10-20$ & 0 & 0 & 2 & 5 \\
\hline $20-30$ & 1 & 5 & 2 & 5 \\
\hline $30-40$ & 5 & 24 & 4 & 10 \\
\hline $40-50$ & 8 & 38 & 12 & 31 \\
\hline $50-60$ & 4 & 19 & 12 & 31 \\
\hline $60-70$ & 3 & 14 & 7 & 18 \\
\hline
\end{tabular}




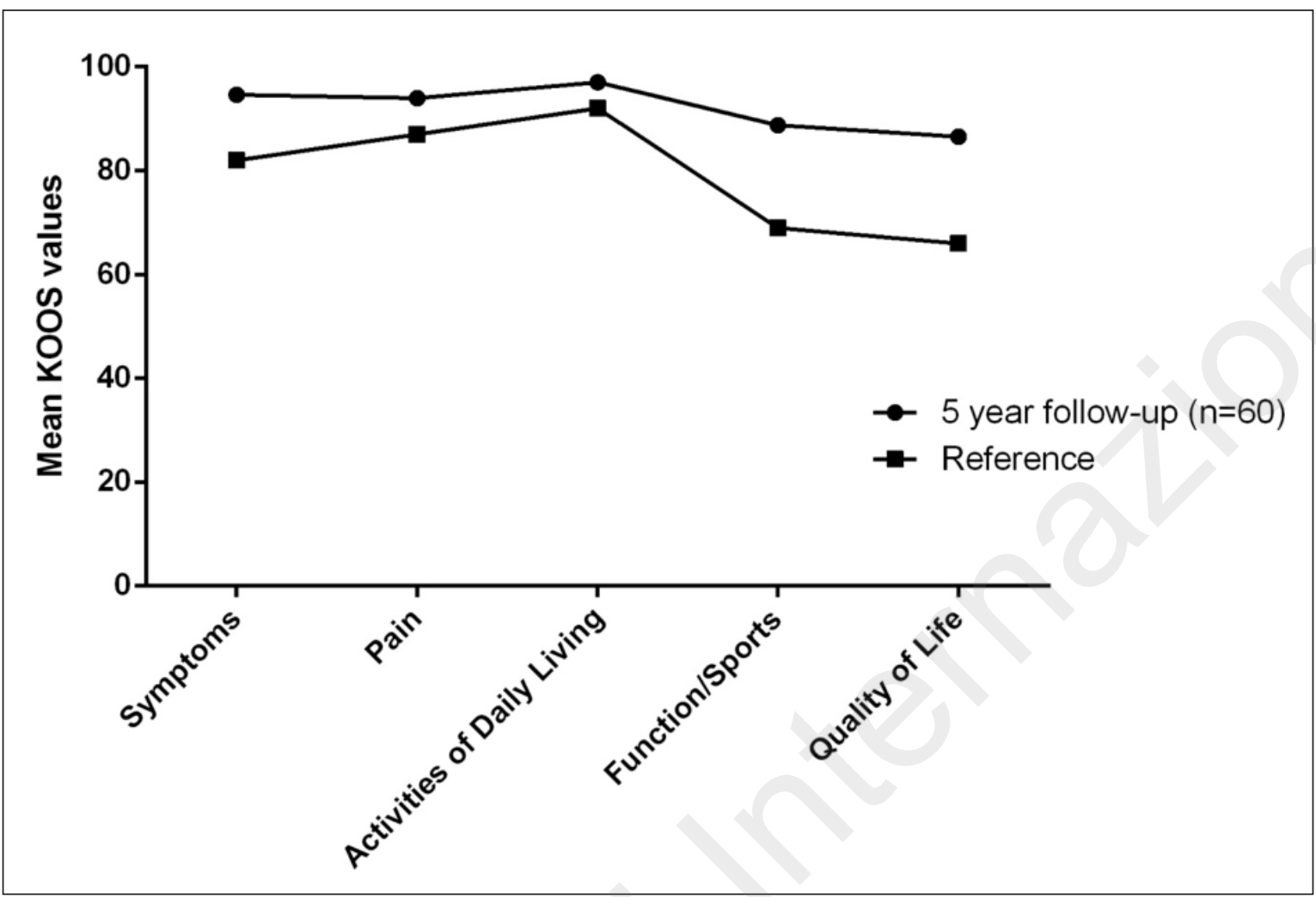

Figure 2. Knee Injury and Osteoarthritis Outcome Score (KOOS) subscores after 5 years minimum follow-up. In comparison to the results of primary ACL reconstruction of a large Scandinavian registry, comparable or higher scores were achieved.

pared to old patients. In the present series, the 2 patients that received revision surgery and ACL reconstruction were 43 and 52 years old when their ACLs were repaired initially. Although this may represent an interesting detail for further consideration, in terms of indication and age limitation, our study population is certainly too small to be able to draw any real conclusions out of this observation.

To our knowledge, the present series is the first in the current literature to report on primary ACL repair with a minimum follow-up of 5 years. There are only a few recent similar studies that describe the clinical results of primary ACL repair. Buechler et al. reported on 45 patients who received an acute ACL repair using a dynamic intraligamentary stabilizing device ${ }^{24}$. Within 12 months, 3 patients had to undergo revision surgery. The median Tegner score was 7 and the median IKDC was 89.5. Objective stability testing revealed excellent results after 12 months. DiFelice et al. presented a series of 11 patients with a minimum followup of two years ${ }^{25}$. In their series, the ACL was repaired with suture anchors that were inserted in the femoral footprint of the ligament's origin. 10 out of the 11 patients had good subjective and clinical results, with a mean subjective IKDC score of 86.4 and an objective IKDC score of $A$ in 9 of 11 patients. The objective stability testing revealed less than $3 \mathrm{~mm}$ side to side difference in all but one patient.
In a systematic review, Taylor et al. pointed out that appropriate patient selection among acute ACL injuries is of crucial importance and should be strictly limited to proximal ruptures with good tissue quality ${ }^{14}$. In our series, 224 out of 1912 patients who received surgical treatment of their ACL injury were selected for primary repair. In our opinion, the here presented ratio of around $11.7 \%$ that qualified for primary repair in our patient population may serve as a guideline or at least as a reference to compare to for future studies. Achtnich et al. presented a study in which 41 out of $420 \mathrm{ACL}$ related surgeries were considered for primary repair $(9.8 \%)^{26} .19$ patients had their $A C L$ repaired within 6 weeks of injury. In the series of DiFelice et al., 11 out of 190 ACL surgeries were performed as primary repair $(5.8 \%)$. Their mean delay from injury to surgery was 39 days (range, 10 to 93), whereas in the present series, all primary repairs were done within 72 hours after the injury. The lower ratio of primary repair may be explained by the longer time to surgery. We consider an early surgical repair as being crucial for this treatment regimen, although the ideal treatment window has not yet been demonstrated.

Obviously, there are some considerable drawbacks in our study. First, apart from the revision rate within five years after surgery, we were only able to report on the subjective outcome following primary ACL re- 
pair. Objective manual or apparative stability testing could not be included in the present series. The main reason for this is based on the fact that our patient population mainly consists of tourists vacating in the neighbouring ski resorts. Given the present encouraging results of a 5-year revision rate that is comparable to that of the conventional $A C L$ reconstruction, it is certainly our intention to include objective stability testing as well as follow-up MRI studies in any future evaluation within the group of patients that can be reached more easily. On the other hand, measuring different aspects of subjective outcome following ACL surgery using the KOOS has been proven to be a very valuable and effective tool in numerous studies. Second, the present questionnaire study had a low response rate of $26.8 \%(60 / 224)$. However, in comparison with other studies that measured patient-reported outcomes after ACL surgery, our response rate was fairly similar. Bojcic et al. compared paper based and electronic surveys following ACL reconstruction using the KOOS 27 . After 5 years their response rate was $20.9 \%$ in the paper-based survey and reached $35.4 \%$ in the electronic version.

\section{Conclusion}

Early primary ACL repair, strictly limited to proximal ruptures with good tissue quality, resulted in a 5-year revision rate that is comparable to $A C L$ reconstruction. Subjective outcome was equal or better than reported for ACL reconstruction. Further studies that include objective stability testing and imaging are certainly needed to assess ligament healing and mechanical integrity.

\section{Conflict of interest}

None of the Authors has any conflict of interest regarding the presented work.

\section{Acknowledgement}

The support by the City of Vienna Competence Team signalTissue project (MA23, \#18-08) is gratefully acknowledged.

\section{References}

1. Strand T, Mølster A, Hordvik M, Krukhaug Y. Long-term followup after primary repair of the anterior cruciate ligament: clinical and radiological evaluation 15-23 years postoperatively. Arch Orthop Trauma Surg. 2005;125(4):217-221.

2. Drogset JO, Grøntvedt T, Jessen V, Tegnander A, Mollnes TE, Bergh K. Comparison of in vitro and in vivo complement activation by metal and bioabsorbable screws used in anterior cruciate ligament reconstruction. Arthroscopy. 2006;22(5):489496.

3. Grøntvedt T, Engebretsen L, Benum P, Fasting O, Mølster A
Strand T. A prospective, randomized study of three operations for acute rupture of the anterior cruciate ligament. Five-year follow-up of one hundred and thirty-one patients. J Bone Joint Surg Am. 1996;78(2):159-168.

4. Bisciotti GN, Quaglia A, Belli A, Carimati G, Volpi P. Return to Sports after ACL reconstruction: a new functional test protocol. MLTJ. 2016;6(4):499-509.

5. Volpi P, Bisciotti GN, Chamari K, Cena E, Carimati G, Bragazz NL. Risk factors of anterior cruciate ligament injury in football players: a systematic review of literature. MLTJ. 2016;6(4): 480-485.

6. Seitz H, Menth-Chiari WA, Lang S, Nau T. Histological evaluation of the healing potential of the anterior cruciate ligament by means of augmented and non-augmented repair: an in vivo animal study. Knee Surg Sports Traumatol Arthrosc. 2008; 16(12):1087-1093.

7. Seitz H, Pichl W, Matzi V, Nau T. Biomechanical evaluation of augmented and nonaugmented primary repair of the anterior cruciate ligament: an in vivo animal study. Int Orthop. 2013;37 (11):2305-2311.

8. Nau T, Teuschl AH. Regeneration of the anterior cruciate ligament: Current strategies in tissue engineering. World J Orthop. 2015;6(1):127-136.

9. Streich NA, Zimmermann D, Bode G, Schmitt H. Reconstructive versus non-reconstructive treatment of anterior cruciate ligament insufficiency. A retrospective matched-pair long-term follow-up. Int Orthop. 2011;35(4):607-613.

10. Oiestad BE, Holm I, Aune AK, et al. Knee function and prevalence of knee osteoarthritis after anterior cruciate ligament reconstruction: a prospective study with 10 to 15 years of followup. Am J Sports Med. 2010;38(11):2201-2210.

11. Proffen BL, Sieker JT, Murray MM. Bio-enhanced repair of the anterior cruciate ligament. Arthroscopy. 2015;31(5):990-997.

12. Koutsari $C$, Snozek $C L H$, Jensen MD. Plasma NEFA storage in adipose tissue in the postprandial state: sex-related and regional differences. Diabetologia. 2008;51(11):2041-2048.

13. Kiapour AM, Fleming BC, Murray MM. Biomechanical Outcomes of Bridge-enhanced Anterior Cruciate Ligament Repair Are Influenced by Sex in a Preclinical Model. Clin Orthop Relat Res. 2015;473(8):2599-2608.

14. Taylor SA, Khair MM, Roberts TR, DiFelice GS. Primary Repair of the Anterior Cruciate Ligament: A Systematic Review. Arthroscopy. 2015;31(11):2233-2247.

15. Padulo J, Oliva F, Frizziero A, Maffulli N. Muscles, Ligaments and Tendons Journal - Basic principles and recommendations in clinical and field Science Research: 2016 Update. MLTJ. 2016;6(1):1-5.

16. Tegner $Y$, Lysholm J. Rating systems in the evaluation of knee ligament injuries. Clin Orthop Relat Res. 1985;(198):43-49.

17. Roos EM, Roos HP, Lohmander LS, Ekdahl C, Beynnon BD. Knee Injury and Osteoarthritis Outcome Score (KOOS)-development of a self-administered outcome measure. J Orthop Sports Phys Ther. 1998;28(2):88-96.

18. Gifstad T, Foss OA, Engebretsen L, et al. Lower risk of revision with patellar tendon autografts compared with hamstring autografts: a registry study based on 45,998 primary ACL reconstructions in Scandinavia. Am J Sports Med. 2014;42 (10):2319-2328.

19. Magnussen RA, Lawrence JTR, West RL, Toth AP, Taylor DC, Garrett WE. Graft size and patient age are predictors of early revision after anterior cruciate ligament reconstruction with hamstring autograft. Arthroscopy. 2012;28(4):526-531.

20. Wright RW, Dunn WR, Amendola A, et al. Risk of tearing the intact anterior cruciate ligament in the contralateral knee and rupturing the anterior cruciate ligament graft during the first 2 years after anterior cruciate ligament reconstruction: a prospective MOON cohort study. Am J Sports Med. 2007; 35(7):1131-1134. 
21. Mariscalco MW, Flanigan DC, Mitchell J, et al. The influence of hamstring autograft size on patient-reported outcomes and risk of revision after anterior cruciate ligament reconstruction: a Multicenter Orthopaedic Outcomes Network (MOON) Cohort Study. Arthroscopy. 2013;29(12):1948-1953.

22. Wright RW, Magnussen RA, Dunn WR, Spindler KP. Ipsilateral graft and contralateral ACL rupture at five years or more following ACL reconstruction: a systematic review. J Bone Joint Surg Am. 2011;93(12):1159-1165.

23. Kvist J, Kartus J, Karlsson J, Forssblad M. Results from the Swedish national anterior cruciate ligament register. Arthroscopy. 2014;30(7):803-810.

24. Büchler L, Regli D, Evangelopoulos DS, et al. Functional recovery following primary $A C L$ repair with dynamic intraliga- mentary stabilization. Knee. 2016;23(3):549-553.

25. DiFelice GS, Villegas C, Taylor S. Anterior Cruciate Ligament Preservation: Early Results of a Novel Arthroscopic Technique for Suture Anchor Primary Anterior Cruciate Ligament Repair. Arthroscopy. 2015;31(11):2162-2171.

26. Achtnich A, Herbst E, Forkel P, Metzlaff S, Sprenker F, Imhoff A, Petersen W. Acute Proximal Anterior Cruciate Ligament Tears: Outcome After Arthroscopic Suture Anchor Repair Versus Anatomic Single-bundle Reconstruction. Arthroscopy. 2016;32(12):2562-2569

27. Bojcic JL, Sue VM, Huon TS, Maletis GB, Inacio MCS. Comparison of paper and electronic surveys for measuring patientreported outcomes after anterior cruciate ligament reconstruction. Perm J. 2014;18(3):22-26. 